SLAC-PUB-8599

August 2000

\title{
IMPEDANCE OF A BEAM TUBE WITH SMALL CORRUGATIONS *
}

\author{
Karl L.F. Bane and G.V. Stupakov \\ Stanford Linear Accelerator Center, Stanford University, Stanford, CA 94309
}

Presented at the XX International Linac Conference (LINAC2000)

Monterey, $C A$

August 21-25, 2000

*Work supported by Department of Energy contract DE-AC03-76SF00515. 


\title{
IMPEDANCE OF A BEAM TUBE WITH SMALL CORRUGATIONS ${ }^{\dagger}$
}

\author{
K.L.F. Bane, G. Stupakov, SLAC, Stanford University, Stanford, CA 94309, U.S.A.
}

\section{INTRODUCTION}

In accelerators with very short bunches, such as is envisioned in the undulator region of the Linac Coherent Light Source (LCLS)[1], the wakefield due to the roughness of the beam-tube walls can have important implications on the required smoothness and minimum radius allowed for the beam tube. Of two theories of roughness impedance, one yields an almost purely inductive impedance[2], the other a single resonator impedance[3]; for smooth bunches, whose length is large compared to the wall perturbation size, these two models give comparable results[4].

Using very detailed, time-domain simulations it was found in Ref. [3] that a beam tube with a random, rough surface has an impedance that is similar to that of one with small, periodic corrugations. It was further found that the wake was similar to that of a thin dielectric layer (with dielectric constant $\epsilon \approx 2$ ) on a metallic tube: $W_{z}(s) \approx$ $2 \mathcal{K}_{0} \cos k_{0} s$, with wave number and loss factor

$$
k_{0}=\frac{2}{\sqrt{a \delta}} \quad \text { and } \quad \mathcal{K}_{0}=\frac{Z_{0} c}{2 \pi a^{2}} ;
$$

with $a$ the tube radius, $\delta$ depth of corrugation, and $Z_{0}=$ $377 \Omega$. For the periodic corrugation problem this result was inferred from simulations for which the period $p \sim \delta$. On the other hand, at the extreme of a tube with shallow oscillations, with $p \gg \delta$, the impedance was found, by a perturbation calculation of Papiernik, to be composed of many weak, closely spaced modes beginning just above pi phase advance[5].

In this report we find the impedance for two geometries of periodic, shallow corrugations: one, with rectangular corrugations using a field matching approach, the other, with smoothly varying oscillations using a more classical perturbation approach. In addition, we explore how these results change character as the period-to-depth of the wall undulation increases, and then compare the results of the two methods.

\section{RECTANGULAR CORRUGATIONS}

Let us consider a cylindrically-symmetric beam tube with the geometry shown in Fig. 1. We limit consideration here to the case $\delta / a$ small; for the moment, in addition, let $\delta / p \gtrsim 1$. We follow the formalism of the field matching program TRANSVRS[6]: In the two regions, $r \leq a$ (the tube region, Region I) and $r \geq a$ (the cavity region, Region II) the Hertz vectors are expanded in a complete, orthogonal set; $E_{z}$ and $H_{\phi}$ are matched at $r=a$; using orthogonality properties an infinite dimensional, homogeneous matrix equation is generated; this matrix is truncated; and finally,

\footnotetext{
${ }^{\dagger}$ Work supported by the U.S. Department of Energy under contract DE-AC03-76SF00515.
}

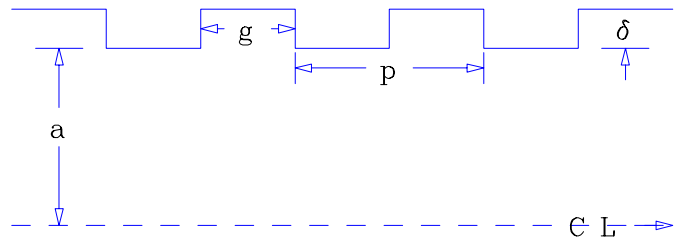

Figure 1: The geometry considered.

the eigenfrequencies are found by setting its determinant to zero. We demonstrate below that, for our parameter regime, the system matrix can be reduced to dimension 1 , and the results become quite simple.

In the tube region, the $z$-component of the Hertz vector

$$
\Pi_{z}^{I}=-\sum_{n=-\infty}^{\infty} \frac{A_{n}}{\chi_{n}^{2}} \frac{I_{0}\left(\chi_{n} r\right)}{I_{0}\left(\chi_{n} a\right)} e^{-j \beta_{n} z},
$$

with $I_{0}$ the modified Bessel function of the first kind, and

$$
\beta_{n}=\beta_{0}+\frac{2 \pi n}{p}, \quad \chi_{n}^{2}=\beta_{n}^{2}-k^{2},
$$

with $\beta_{0}$ the phase advance and $k$ the wave number of the mode. In the cavity region,

$$
\begin{gathered}
\Pi_{z}^{I I}=-\sum_{s=0}^{\infty} \frac{C_{s}}{\Gamma_{s}^{2}} \frac{R_{0}\left(\Gamma_{s} r\right)}{R_{0}\left(\Gamma_{s} a\right)} \cos \left[\alpha_{s}(z+g / 2)\right], \\
\alpha_{s}=\frac{\pi s}{g}, \quad \Gamma_{s}^{2}=\alpha_{s}^{2}-k^{2} \\
R_{0}\left(\Gamma_{s} r\right)=K_{0}\left(\Gamma_{s}[a+\delta]\right) I_{0}\left(\Gamma_{s} r\right)-I_{0}\left(\Gamma_{s}[a+\delta]\right) K_{0}\left(\Gamma_{s} r\right),
\end{gathered}
$$

with $K_{0}$ the modified Bessel Function of the second kind.

$E_{z}$ and $H_{\phi}$ are given by

$$
E_{z}=\left(\frac{\partial^{2}}{\partial z^{2}}+k^{2}\right) \Pi_{z}, \quad Z_{0} H_{\phi}=-j k \frac{\partial \Pi_{z}}{\partial r} .
$$

Matching these fields at $r=a$, and using the orthogonality of $e^{-\beta_{n} z}$ on $[-p / 2, p / 2]$, and $\cos \left[\alpha_{s}(z+g / 2)\right]$ on $[-g / 2, g / 2]$ we obtain a homogeneous matrix equation. To find the frequencies, the determinant is set to zero; i.e.

$$
\operatorname{det}\left[\mathcal{R}-\left(\frac{2 g}{p}\right) N^{T} \mathcal{I} N\right]=0,
$$

with the matrix $N$ given by

$$
N_{n s}=\frac{2 \beta_{n}}{\left(\beta_{n}^{2}-\alpha_{s}^{2}\right) g}\left\{\begin{array}{lll}
\sin \left(\beta_{n} g / 2\right) & : & s \text { even } \\
\cos \left(\beta_{n} g / 2\right) & : & s \text { odd }
\end{array},\right.
$$

and the diagonal matrices $\mathcal{R}$ and $\mathcal{I}$ by

$$
\mathcal{R}_{s}=\left(1+\delta_{s 0}\right) k a\left(\frac{R_{0}^{\prime}}{x R_{0}}\right)_{\Gamma_{s} a}, \quad \mathcal{I}_{n}=k a\left(\frac{I_{0}^{\prime}}{x I_{0}}\right)_{\substack{\chi_{n} a \\(10)}} .
$$


For the beam, on average, to interact with a mode, one space harmonic of the mode must be synchronous. We will pick the $n=0$ space harmonic to be the synchronous one; i.e. let $\beta_{0}=k$ (we take the particle velocity to be $v=$ c). We truncate the system matrix to dimension 1, keeping only the $n=0$ and $s=0$ terms in the calculation (the other terms are small). Now if $k \delta$ is small, then the $s=0$ term in $\mathcal{R}$ becomes $\mathcal{R}_{0}=2 /(k \delta)$, the $n=0$ term in $\mathcal{I}$ is $\mathcal{I}_{0}=k a / 2$, and $N_{00} \approx 1$. Eq. 8 then yields

$$
k=\sqrt{\frac{2 p}{a \delta g}},
$$

which, for $p=2 g$, equals $k_{0}$ of Eq. 1 .

The loss factor is given by $\mathcal{K}=|V|^{2} /\left[4 U p\left(1-\beta_{g}\right)\right][7]$, with $V$ the voltage lost by the beam to the mode, $U$ the energy stored in the mode, and $\beta_{g}$ the group velocity over $c$. The voltage lost in one cell is given by the synchronous $(n=0)$ space harmonic: $V=A_{0} p$, and the energy stored in one cell, $U=1 /\left(2 Z_{0} c\right) \int E \cdot E^{*} d v$, is approximately that which is in the $n=0$ space harmonic: $U=\pi A_{0}^{2} a^{2} p\left(1+k^{2} a^{2} / 8\right) /\left(2 Z_{0} c\right)$ (for details, see Ref. [6]). For $\beta_{g}$, we take Eq. 8 truncated to dimension 1 , and expand near the synchronous point. Taking the derivative with respect to $\beta_{0}$ and then setting $\beta_{0}=k$ we obtain:

$$
\left(1-\beta_{g}\right)=\frac{4 \delta g}{a p} .
$$

The loss factor becomes $\mathcal{K}=\mathcal{K}_{0}$.

The above method can be extended to modes of higher multipole moment $m$, in which case the beam will excite hybrid modes rather than the pure TM modes of above[6]. Again the system matrix can be reduced to the $n=0$ and $s=0$ terms, and the lowest mode wave number and loss factor have a simple form (for $1 \leq m \ll a / \delta$ ):

$$
k=\sqrt{\frac{(m+1) p}{a \delta g}} \text { and } \mathcal{K}=\frac{Z_{0} c}{\pi a^{2(m+1)}},
$$

and $\left(1-\beta_{g}\right)=m(m+2) \delta g /(a p)$. We note that the dipole $(m=1)$ frequency is equal to the monopole $(m=0)$ frequency. Also, the wake at the origin is the same as for the resistive-wall wake of a cylindrical tube[8], as we expect.

Running TRANSVRS with a matrix of dimension 40, we obtain a typical dispersion curve (see Fig. 2). Here $k / k_{0}=1.07, \mathcal{K} / \mathcal{K}_{0}=.94$. Note that even when $\delta / a$ is not so small, e.g. for bellows with $\delta / a \approx .2[9]$, the analytical formulas are still useful. Fig. 3 shows how the strength and frequency of the mode change as the period of undulation is increased. The scale over which $\mathcal{K}$ drops to zero is $p_{0} \approx \pi \sqrt{a \delta g / 2 p}$. By $p \sim p_{0}$, the one dominant mode has disappeared, and we are left with the many weak, closely spaced modes, beginning just above $k p=\pi$, that were found by Papiernik.

\section{SINUSOIDAL CORRUGATIONS}

Let us assume now that the pipe surface is given by

$$
r=a-h \sin \kappa z,
$$

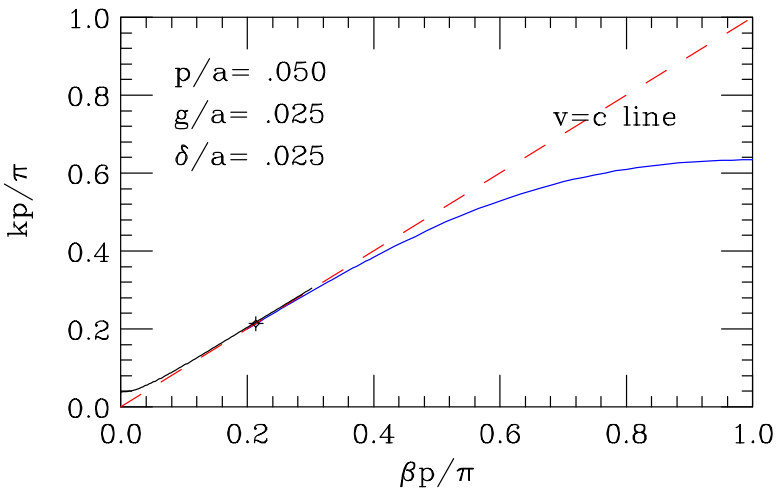

Figure 2: Dispersion curve example.

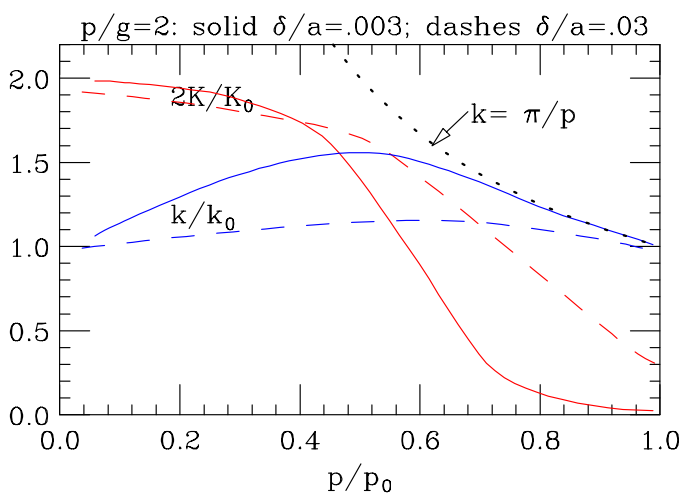

Figure 3: An example showing the effect of varying $p$.

where $2 \pi / \kappa$ is the period of corrugation, and $h$ is its amplitude. We assume that both the amplitude and the wavelength are small, $h \ll a$ and $\kappa a \gg 1$. This allows us to neglect the curvature effects and to consider the surface locally as a plane one. We will also assume a shallow corrugation $h \kappa \ll 1$, i.e. the amplitude of oscillation is much smaller than the period.

Introducing a local Cartesian coordinate system $x, y, z$ with $y=a-r$ (directed from the wall toward the beam axis), and $x$ directed along $\phi$, the surface equation becomes $y=y_{0}(z) \equiv h \sin \kappa z$. The magnetic field near the surface $H_{x}(y, z)$ does not depend on $x$ (that is $\phi$ ) due to the axisymmetry of the problem. It satisfies the Helmholtz equation

$$
\frac{\partial^{2} H_{x}}{\partial y^{2}}+\frac{\partial^{2} H_{x}}{\partial z^{2}}+k^{2} H_{x}=0
$$

with the boundary condition

$$
\left.(\vec{n} \nabla H)\right|_{y=y_{0}}=0,
$$

where $\vec{n}$ is the normal vector to the surface, $\vec{n}=$ $(0,1,-h \kappa \cos \kappa z)$.

Note that the longitudinal electric field $E_{z}$ can be expressed in terms of $H_{x}$,

$$
E_{z}=-\frac{i}{k} \frac{\partial H_{x}}{\partial y} .
$$


Using the small parameter $h / a$, we will develop a perturbation theory for calculation of $H_{x}$ near the surface and find how $E_{z}$ is related to $H_{x}$.

In the zeroth approximation, the $z$ dependence of $H_{x}$ is dictated by the beam current periodicity,

$$
H_{x}(y, z)=\mathcal{H}(y) e^{i k z} .
$$

Putting Eq. (18) into Eq. (15) we find that $d^{2} \mathcal{H} / d y^{2}=0$, hence $\mathcal{H}(y)=H_{0}+A y$, where the constant $A$ can be related, through Eq. (17), to the electric field on the surface, $A=i k E_{z}$. We will see below that $A$ is second order in $h$.

For a flat surface, for which $\vec{n}=(0,1,0)$, from the boundary condition (16), we would conclude that $A=0$, however, the corrugations result in a nonzero $A$, and hence $E_{z}$. Substituting the magnetic field (18) into the right hand side of Eq. (16) one finds

$\vec{n} \nabla H=-\frac{1}{2} i h k \kappa H_{0}\left[e^{i(k+\kappa) z}-e^{i(k-\kappa) z}\right]-i k \zeta H_{0} e^{i k x}$.

Clearly, the boundary condition is not satisfied in this approximation. To correct this, we have to add satellite modes to the fundamental solution (18)

$$
\begin{gathered}
H_{x}(y, z)=\mathcal{H}(y) e^{i k z}+\mathcal{H}_{1}(y, z), \\
\mathcal{H}_{1}(y, z)=B^{+}(y) e^{i(k+\kappa) z}+B^{-}(y) e^{i(k-\kappa) z} .
\end{gathered}
$$

The dependence of $B^{ \pm}$versus $y$ can be found from the Helmholtz equation,

$$
B=B_{0}^{ \pm} e^{-y \sqrt{\kappa^{2} \pm 2 \kappa k}},
$$

where $B_{0}^{ \pm}$are constants. In order for $B^{ \pm}$to exponentially decay in $y$, we have to assume here that $k<\kappa / 2$.

Substituting $\mathcal{H}_{1}$ terms into the boundary condition (16) generates first order terms that have $x$-dependence $\exp i(k \pm \kappa) x$, and second order terms proportional to $\exp (i k x)$. From the former one finds that

$$
B_{0}^{ \pm}=-\frac{i k \kappa H_{0} h}{2 \sqrt{\kappa^{2} \pm 2 k \kappa}},
$$

and the latter gives an expression for the tangential electric field on the surface,

$$
E_{z}=\frac{1}{4} i k h^{2} \kappa H_{x} \frac{\sqrt{\kappa^{2}+2 k \kappa}+\sqrt{\kappa^{2}-2 k \kappa}}{\sqrt{\kappa^{2}-4 k^{2}}} .
$$

One can now solve Maxwell's equations with the boundary condition given by Eq. (23) (see details in [10]). It turns out, that in the region of frequencies $k<\kappa / 2$ there exists a solution corresponding to a wave propagating with the phase frequency equal to the speed of light. The frequency and the loss factor of the mode are shown in Fig. 4 (solid lines). We see that decreasing the height of the corrugation results in smaller wakes, and hence leads to the suppression of the interaction of the synchronous wave with the beam. In the limit of small frequencies, $k \ll \kappa$ the frequency is

$$
k_{1}=\frac{2}{h \sqrt{a \kappa}} \text {. }
$$

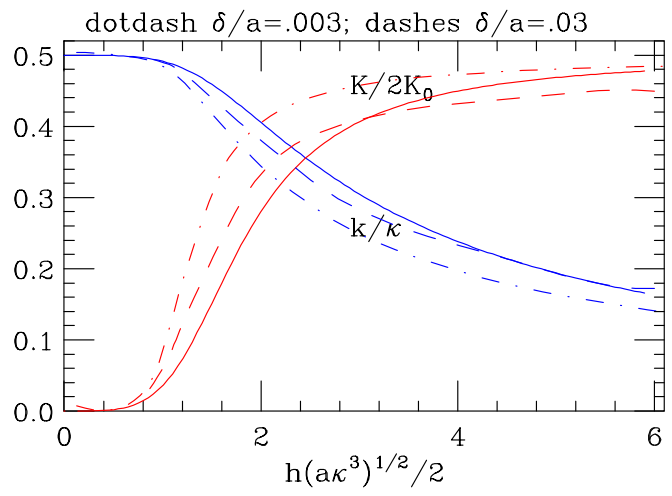

Figure 4: Frequency and loss factor as function of height.

We should mention here that the perturbation theory breaks down for very small values of $h$. Indeed, we implicitly assumed that the satellite harmonics in Eq. (21) are localized near the surface, otherwise our approximation of plane surface becomes invalid. Hence, we have to require that $k-2 k \gg a^{-1}$, which gives the following condition of applicability: $h>a^{-1 / 4} \kappa^{-5 / 4}$. This condition explains why this mode was not found by Papiernik: being perturbative in parameter $h$ the approach developed in his paper is applicable only when $h$ can be made arbitrarily small. Let us also mention that our approach can be applied to the rectangular corrugations problem: we take Faraday's law, to obtain the averaged longitudinal field at $r=a,\left\langle E_{z}\right\rangle=i k \delta g H_{x} / p$, and then follow the method of Ref. [10] to reproduce Eq. 11.

Finally, in Fig. 4 we include also the results of Fig. 3, obtained by field matching (the dashes, the dotdashes). For the comparison we make the correspondences with the first Fourier component of the wall shape: $\kappa=2 \pi / p$ and $h=2 \delta / \pi$. We note that even though the rectangular corrugations violate our requirement for smoothness, the results for the two methods are very similar.

Acknowledgement: We thank A. Novokhatskii for his contribution to our understanding of the problem of roughness impedance.

\section{REFERENCES}

[1] Linac Coherent Light Source (LCLS) Design Study Report. SLAC-R-521, Apr 1998.

[2] K. Bane, et al, PAC97, p. 1738 (1997); G.V. Stupakov, Phys. Rev. AB 1, 64401 (1998).

[3] A. Mosnier and A. Novokhatskii, PAC97, p. 1661 (1997).

[4] K. Bane and A. Novokhatskii, SLAC-AP-177, March 1999.

[5] M. Chatard-Moulin and A. Papiernik, IEEE Trans. Nucl. Sci. 26, 3523 (1979).

[6] K. Bane and B. Zotter, Proceedings of the $11^{\text {th }}$ Int. Conf. on High Energy Accelerators, CERN, p. 581 (1980).

[7] See, e.g., E. Chojnacki, et al, PAC93, p. 815, 1993; A. Millich, L. Thorndahl, CERN-CLIC-NOTE-366, Jan. 1999.

[8] A. Chao, "Physics of Collective Instabilities in High-Energy Accelerators", John Wiley \& Sons, New York (1993).

[9] K. Bane and R. Ruth, SLAC-PUB-3862, January 1986.

[10] G.V. Stupakov in T. Roser and S. Y. Zhang, eds., AIP Conference Proceedings 496, 1999, p. 341. 